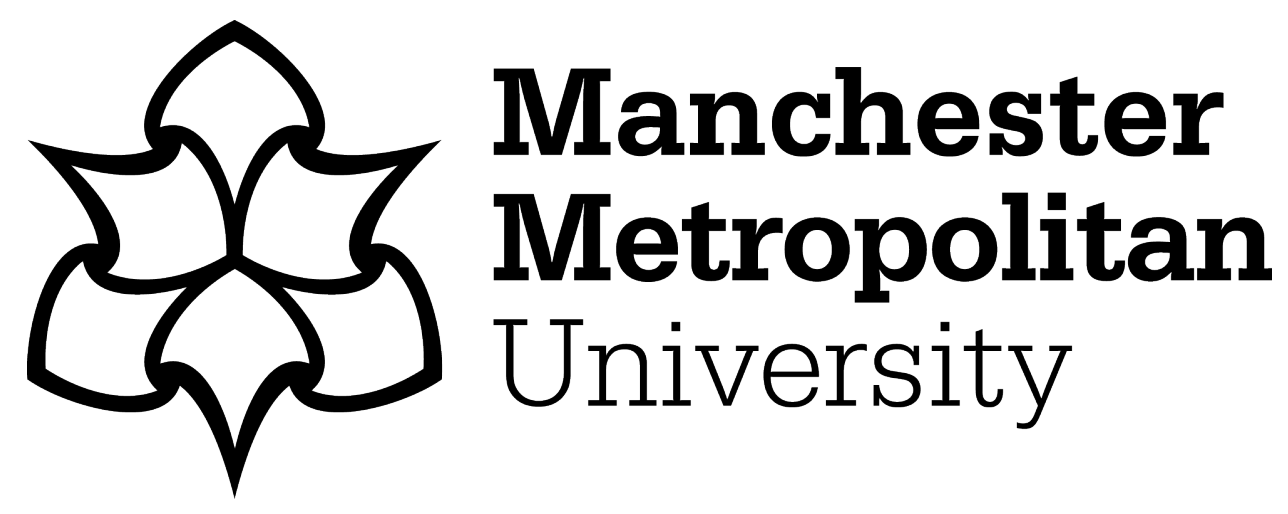

Osman, O and Yap, Moi (2019) Computational Intelligence in Automatic Face Age Estimation: A Survey. IEEE Transactions on Emerging Topics in Computational Intelligence, 3 (3). pp. 271-285. ISSN 2471-285X

Downloaded from: https://e-space.mmu.ac.uk/621398/

Version: Accepted Version

Publisher: IEEE

DOI: https://doi.org/10.1109/TETCI.2018.2864554

Please cite the published version 


\section{Author Copy}

Please cite:

O. F. Osman and M. H. Yap (2018), "Computational Intelligence in Automatic Face Age Estimation: A Survey," IEEE Transactions on Emerging Topics in Computational Intelligence, Vol. 2, issue 5.

doi: $10.1109 /$ TETCI.2018.2864554

@ 2018 IEEE. Personal use of this material is permitted. Permission from IEEE must be obtained for all other uses, in any current or future media, including reprinting/republishing this works, for resale or redistribution to servers or lists, or reuse of any copyrighted component of this work in other works. 


\title{
Computational Intelligence in Automatic Face Age Estimation: A Survey
}

\author{
Omaima FathElrahman Osman and Moi Hoon Yap, Member, IEEE
}

\begin{abstract}
With the rapid growth of computational intelligence techniques, automatic face age estimation has achieved good accuracy that benefited real-world applications such as access control and monitoring, soft biometrics and information retrieval. Over the past decade, many new algorithms were developed and previous surveys on face age estimation were either outdated or incomplete. Considering the importance of the expanding research in this topic, we aim to provide an up-to-date survey on the face age estimation techniques. First we summarise the stateof-the-art databases and the performance metrics for face age estimation. Then we review the age estimation techniques based on three categories of face features (local, global and hybrid) and discuss different types of age learning algorithms. Finally, we identify the challenges and provide new insights for future research directions of fully automated face age estimation.
\end{abstract}

Index Terms-Age estimation, face features, feature extraction, age learning, machine learning.

\section{INTRODUCTION}

$\mathbf{I}$ $\mathrm{N}$ human communication, face is the first source that provides information to identify a person (such as age, gender and ethnicity), to facilitate communication (mood and emotion) and to explore the skin condition of a person (cosmetic and social habit). Researchers have developed face analysis applications such as face recognition [1], [2], facial expression recognition [3], gender classification [4], face age estimation [5], [6], and facial skin assessment [7], [8]. Amongst these applications, face age estimation has received considerable attention as age can enhance the performance of face recognition, facilitate communication and affect skin quality or appearance.

Face age estimation is defined as the possibility to "label a face image automatically with the exact age (eg. 20 year) or the age group (eg. young, adult) of the individual face" [5]. The goal of automatic age estimation is to judge if age is as close to actual or appearance age as possible. Actual or real age is the number of years that a person has lived while appearance age is the age information revealed by the visual appearance. Perceived and estimated age is the individual age recognized by human and computer respectively from the visual appearance [9]. Age estimation is useful in many real-world applications such as Access Control and

O.F. Osman at the College of Computer Science and Information Technology, Sudan University for Science and Technology, Khartoum, Sudan. Email: omaima.osman2012@gmail.com.

M.H. Yap is with the School of Computing, Mathematics and Digital Technology, Manchester Metropolitan University, M1 5GD, Manchester, UK. E-mail: m.yap@mmu.ac.uk.

This is author copy, please download the publisher version at https://ieeexplore.ieee.org/document/8443121/.
Monitoring, Soft Biometric, Electronic Customer Relationship Management and Entertainment.

In Access Control and Surveillance Monitoring, an accurate age estimation system can prevent children from entering to unauthorized places or websites, when a monitoring camera is used with the system [5], and prevent child and adult seniors from using danger games in a theme park [10]. To control access in a cigarette vending machine, a Japanese company developed vending machine that check a user age by counting wrinkles and skin sags to prevent anyone under the legal age (20 years old) from buying a cigarette[11]. Age estimation system also can be used in health care systems for customized services such as robotic nurse and intelligent intensive care unit [5]. Age estimation as a soft biometrics can be used to improve the recognition accuracies in basic biometric system [12].

The effective marketing strategy which called Electronic Customer Relationship Management (ECRM) [5] can benefit from age estimation systems by targeting specific customers in same age group for specific advertisements [13]. It can provide important information for marketing study such as the number of young and adult customers who have visited a mall along with the preferences of each age group [14]. However, it is difficult to access this information while retaining privacy, but using automatic age estimation system can help to perform this task readily without violating the human privacy as the face image of a customer can be captured to identify age and delete the images instantly [5].

Recently, age estimation system is introduced as an application in some smart phones for Entertainment purposes such as iPhone Age Detector. When many photos and videos are captured, age information can organize the images to make them easy to access and find the related images when needed (Automatic album management) [14]. This also can be used for age-based image and video retrieval systems (information retrieval), where users could have the ability to restore their images by determining a wanted age-group in e-photo albums.

Age estimation also can be used with age synthesis in Forensic Art to find lost people. When the photos of missing children or any other family members are outdated, the system can predict the age and use age synthesis to produce updated face image [15]. In Human Computer Interaction (HCI) systems, computer interface can use a face image, classify the age and setup a workspace automatically according to the user's age group [5].

Although there are several techniques that are used in stateof-the-art algorithms to solve age estimation problem, it is yet largely unsolved and remained a challenging issue for the 
following reasons:

1) Age estimation is affected mainly by human aging process, including different changes in human face. In addition to these normal changes, there are many external factors such as environmental influence, solar radiation, lifestyle, disease, drug use, and psychological stress that can have effect on face aging process and make it uncontrollable and personalized process [16].

2) The existing input image variations such as different illumination, pose and facial expressions can have a significant impact on age estimation system performance [17].

3) It is difficult to collect a large-scale longitudinal face age dataset for detailed aging progression [5].

Hence, a robust method to represent face image and extract the discriminative features that reduce the negative influence of personalized property is still needed. Although there are several age estimation surveys, e.g. Fu et al. [5] in 2010, Guo and Guodong [14] in 2012 and Dantcheva et al. [12] in 2016, they have not included the new and emerging approaches that significantly increase the accuracy of age estimation. The most recent survey by Dantcheva et al. [12] focused on different soft biometrics attributes, but the age estimation survey is dated as it was mainly based on the 2010 survey by Fu et al. [5]. To bridge the gap, this paper provides a comprehensive survey on automatic face age estimation. It particularly reviews the computational intelligence techniques in the state-of-theart algorithms and discusses the potential technique, including deep learning, to improve the performance of automatic face age estimation.

This paper is structured as follows: Section 2 discusses different types of face age databases that commonly used in state-of-the-art algorithms and its limitations. Section 3 presents the performance metrics for age estimation. Section 4 surveys the state-of-the-art age estimation algorithms in two phases: feature types and learning algorithms. Finally, conclusions and future directions are summarized in Section 5.

\section{Face Aging Database}

There are different types of datasets used in previous work on face age estimation such as CACD [26], FERET [27], FGNET [28], MORPH [29], [38], PAL [30], YGA [31], [39], WIT [32], HOIP [34], [5], AI \&R [5], [33], IAD [37], Iranian Face Database [35] and LHI [36]. However, most of these datasets are not publicly available. The available datasets that provide a large amount of face image with accurate age information are FG-NET [28], FERET [27], MORPH [29], PAL [30], CACD [26] and Iranian face dataset (only male images are available) [35]. The overall comparison of the face aging databases is illustrated in Table I. This section discusses the strength and limitations of the publicly available databases, i.e. FG-NET, MORPH, FERET, PAL and CACD.

FG-NET [28] is a popular baseline database that benchmarks the performance of the existing age estimation algorithms with new algorithms. It contains images for infants and a series of images for individuals during different age

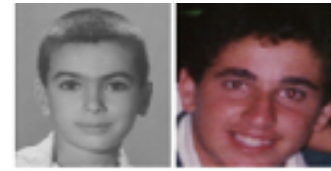

M 08

$$
\text { M } 21
$$
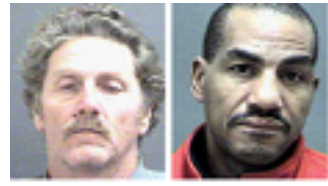

M 51

M 39

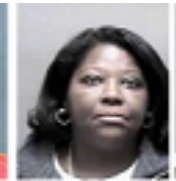

F 46

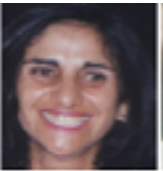

F 30

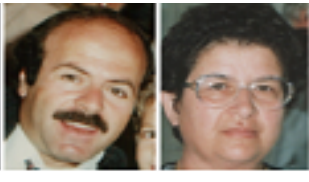

M 31

$F 50$
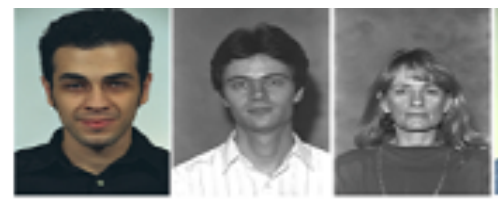

M 50

F 44

M 21

M 30

F 40
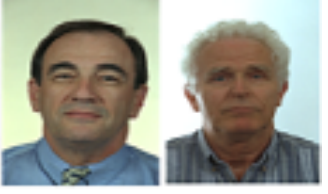

M 62
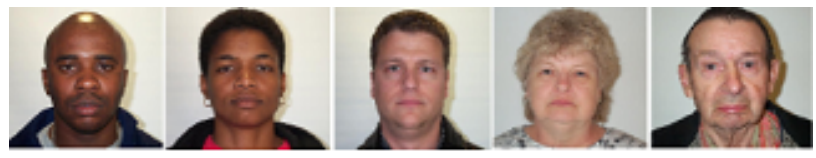

M 26

F33

M 40

F 60

M 88
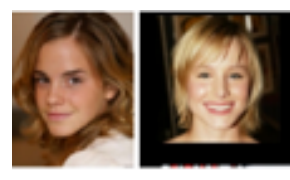

F 24

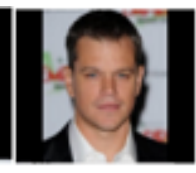

M 38
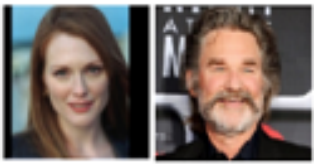

M 62

Fig. 1. Sample face images from the publicly available benchmark databases Row 1: FG-NET [28]; Row 2: MORPH [29]; Row 3: FERET [27]; Row 4: PAL [30]; and Row 5: CACD [26]. These databases are varied in pose, expression, lighting and image resolution.

progression stages. However, FG-NET images were collected by scanning photographs, thus there were very large variations in resolution, background, illumination and noise from scanner. This might complicate the detecting of texture information such as wrinkles.

MORPH [29] is a benchmark database contains 55,134 images, a relatively large-scale database if compared to FG-NET. It was a collection of mugshot images, including information about ethnicity, gender, date of birth and date of acquisition. 42,589 images are African, others are European, Asian and Hispanic.

FERET [27] database were collected in a controlled environment and the image resolution is better if compared to FG-NET and MORPH. It has more representative texture information that works better for local descriptor extraction.

PAL [30] database contains wide age range for adulthood. It contains images for subject with maximum age of 93 years old, which is important for research on aging. The images were captured with good resolution.

CACD [26] is the largest publicly available databases used recently in age estimation, face recognition and retrieval 
TABLE I

A SUMMARY OF FACE AGING DATABASES. IT COMPARES AND ILLUSTRATES THE LIMITATIONS OF THE EXISTING DATABASES INCLUDING INCONSISTENCIES ON THE NUMBER OF IMAGES PER SUBJECT, AGE RANGE, AGE TYPE AND ETHNICITY.

\begin{tabular}{|c|c|c|c|c|c|c|c|}
\hline Name & Total Images & Total Subjects & Age Range & Age Type & Status & Ethnicity & Expression \\
\hline CACD [26] & 163,446 & 2,000 & $16-62$ & Exact age & Public & Yes & Yes \\
\hline FERET [27] & 14,126 & 1199 & $10-70$ & Exact age & Public & Yes & Yes \\
\hline FG-NET [28] & 1,002 & 82 & $0-69$ & Exact age & Public & Yes & Yes \\
\hline MORPH [29] & 55,134 & 13,000 & $16-77$ & Exact age & Public & Yes & Yes \\
\hline PAL [30] & 1,142 & 576 & $18-93$ & Age Range & Public & Yes & No \\
\hline YGA [31] & 8000 & 1600 & $0-93$ & Exact age & Private & Asian & Yes \\
\hline WIT [32] & 26,222 & 5,500 & $3-85$ & Age Range & Private & Japanese & No \\
\hline AI\&R (V2.0) [33] & 34 & 17 & $22-61$ & Age Range & Private & Asian & yes \\
\hline HOIP [34] & 306,600 & 300 & $15-64$ & Age Range & Private & Japanese & No \\
\hline Iranian [35] & 3,600 & 616 & $1-90$ & Exact age & Private & Middle-East & Yes \\
\hline LHI [36] & 8,000 & 8,000 & $9-89$ & Exact age & Private & Asian & no \\
\hline IAD [37] & 219,892 & - & $1-80$ & Exact age & Private & Yes & Yes \\
\hline
\end{tabular}

across age research. It contains images for a large number of celebrities collected from internet across ten years. However, this dataset does not include face images with age of 10 or younger. Even though internet is a good platform for data collection, it is very hard to label the ground truth of age or it might not be accurate. In addition, the data collected from the internet could be chaotic.

Although MORPH and CACD databases have large number of images, FERET and PAL with better resolution, they do not contain face images in childhood. Other limitations of the existing databases is the inclusion of certain ethnicities, i.g. YGA, AI\&R. Also, the LHI are datasets consist of Asian subjects while WIT contains only Japanese. On the other hand, capturing face images at different ages for the same subject is a big challenge. Besides, we observe that there is lack of consistency and standardisation in data collection, e.g., the age range and the number of images per subject. In some databases such as YGA, MORPH and LHI, only one or a small number of images for each subject is captured, while FG-NET consists of 10 images per subject. WIT captures 1 - 14 image samples from each individual. AI\&R database includes limited number of face images. According to Guo et al. [14], databases with a limited number of face images of each subject might not be ideal for the age progression algorithms.

Although there are a number of datasets for age estimation, a large database with balanced distribution of individuals, ethnicity, gender, and age range is still needed. Figure 1 shows the variation in a different conditions such as pose, expression, lighting and makeup in publicly available databases, and Table I provides a summary and a comparison of different face aging databases.

\section{Performance Metrics For Age Estimation}

Two popular metrics are widely used in the literature [40], [41], [42], [43] to measure the performance of age estimation algorithms, i.e. Mean Absolute Error (MAE) and Cumulative Score (CS). MAE is the average of absolute errors between estimated age and the ground truth [44], it is commonly used in previous age estimation research to measure the accuracy of an algorithm. The mathematical form of MAE is shown in equation (1).

$$
M A E=\frac{1}{X} \sum_{n=1}^{X}\left|a_{n}^{\prime}-a_{n}\right|
$$

where $\mathrm{X}$ is the number of images in the test set, $a_{n}$ is the n-th ground truth, and $a_{n}^{\prime}$ is the estimated age [45]. In addition to general MAE, Guo et al. [46] used "MAE per age" and "MAE per decade" to show the performance of age estimation in more details and allow to compare different approaches to show which method produces better result at each decade or age [41], [46].

The CS measure illustrates the age estimation accuracy by drawing curves in various acceptable error levels and can be defined as:

$$
C S(I)=X_{e \leq I} / X \times 100 \% .
$$

where $X_{e \leq I}$ is the total number of test images the age estimation produce an absolute error less than $I$ years [41].

\section{Face Age Estimation Techniques}

This section contains a survey on face age estimation techniques using computational intelligence algorithms. The state-of-the-art age estimation algorithms predict the exact age or to identify the age range such as teenager or child [47]. Most of these algorithms are composed of two phases: features extraction and age learning [47], [5], [48].

\section{A. Features Extraction}

The performance of age estimation system is affected mainly by features extracted from face images. Feature extraction means the face features related to facial appearance changes caused by aging extracted from human face to represent the age [49].

Facial features can be classified as local, global, and hybrid. Local and global features have different roles in face perception. Both of them contains a discriminative data for face analysis. Local information is embedded in the detailed 
local variations of the facial appearance, while global information means the overall structural configuration of the facial contour[50]. As such, facial features are responsible for corresponding classification of age estimation algorithms local-based, global-based and hybrid. Table II summarizes the published work in age estimation using different types of features.

1) Local-Based Algorithms: Local features include the geometry of facial components including age spots, depth, wrinkles, and freckles. Local features are usually used in age groups classification. i.e., infant, young and adults, due to the unique characteristics found on local features to differentiate between age groups. For example, the changes in distance ratios between eyes, nose, and mouth are shown clearly in babyhood, while wrinkles are more notable in adulthood. Thus, these features are more suitable to classify age into groups [40].

The first publication on face age estimation was by Kwon and Lobo [51], [52]. They outlined a computational theory for age classification in which they used a combination of distance ratios between facial parts and wrinkle analysis to classify people into children, young adults, and senior adults. They consult studies in cranio-facial research and suggest that the revised cardioidal strain transformation model is the best model to describe human head growth. To differentiate baby faces from the two older groups, a set of distance ratios was computed between the face parts (mouth, nose, forehead, chin, and eyes). Snakelets analysis are used to find wrinkles to differentiate young adults from senior adults. They used only 47 face images in their experiment [52].

Since the methods used by Kwon and Lobo [51], [52] for localization are computationally expensive and database that they used is small [53], Horng et al. [54] proposed age classification algorithm that classify the age into four groups: children, young, middle-aged adults, and senior adults. Firstly, they applied Sobel edge operator to define the positions of eyes, noses, and mouths. To extract the features from the face, two geometric features were used as distances ratios between eyes, noses, and mouths to distinguish babies from other groups. The degree of wrinkles from five areas (forehead, two cheeks, and two eye corners) was found using Sobel edge magnitudes to differentiate between young adults, middle-aged adults, and senior adults. Finally, two back-propagation neural networks were constructed to classify the age. The first one was employed to distinguish the baby face from other classes using geometric features. If it is not a baby, then the second network was used to differentiate between the others three adult groups based on wrinkle features. They achieved $81.58 \%$ identification rate using 115 gray-level images [54].

In 2006, Ramanathan and Chellappa [55] proposed a craniofacial growth model to estimate the age during formative years (0 - 18 years). They described the growth of a face using growth parameters that defined over facial landmarks commonly used in anthropometric studies. They detected facial features from eyes, mouth and the outer contour of the face by fitting ellipses of various sizes and orientations.

However, the basic model used by [51], [52], [54], [55], known as anthropometric model, consider the geometry infor- mation only rather than texture information to be appropriate for identifying young age group. It is not useful for the identification of the adults because most changes pertaining to them appear as texture, so that in [51], [54] the authors used wrinkles analysis in addition to anthropometric model to extract aging features. Anthropometric model is sensitive to head pose in that only front face can be used to measure facial geometries. The model also is inappropriate to predict the age in detail [48], [5].

In computer vision, the biologically inspired model [56] have shown superior results in some tasks such as face recognition [1] and object category recognition [57]. The model in [56] consists of simple (S) and complex (C) layers alternately. $S$ units are created using Gabor filters at four orientations and 16 scales. There are 8 bands of units for each orientation created by grouping the adjacent two scales of $\mathrm{S}$ units together. Then $\mathrm{C}$ units were generated by taking the maximum values within a local spatial neighborhood and across the scales within a band. The MAX operator is used as pooling filter in $\mathrm{C}$ units [56].

In 2009 Guo et al. [41] introduced biologically inspired model in age estimation task for the first time and called it Biologically Inspired Feature (BIF). They applied the model in [56] with some improvements in $\mathrm{C}$ units generated using nonlinear operations including the MAX operation and an standard deviation (STD) operation to obtain a high-performance. Both of these processes were done independently for every orientation and scale band. For every face image, a feature vector was constructed by concatenating the outputs of $\mathrm{C}$ units. After extracting the biologically inspired features in $\mathrm{C}$, Principal Component Analysis (PCA) was applied for dimensionality reduction while Support Vector Machine (SVM) was applied for age learning. Although the BIF showed a significant improvements in age estimation accuracy, it represent the face images in a high dimension feature vector [14].

Ylioinas et al.[58] proposed an age estimation model using Kernel Local Binary Pattern (KLBP) to represent facial features. Firstly, the face was aligned using similarity transformation and the face pose was corrected using an flipping operation to evaluate the pose correction method. For feature extraction the method compared between a histogram and a kernel density estimate for LBP. Finally, a Support Vector Regression (SVR) was applied for age estimation. Ylioinas et al.[58] found that the kernel method was more powerful than the histogram using pose corrected images, which achieved 5.09 and 5.23 respectively for MAE. Using the original images, the MAE is 5.20 for kernel and 5.39 for histogram, which means that the pose correction enhanced the age estimation performance [58].

In 2013 Gunay et al. [59] also extracted local features to estimate the age using Radon transform. After the preprocessing step which included cropping, scaling and resize images to $88 \times 88$, The Radon transform was applied for an image matrix $f(x, y)$ to compute the projections in specified direction. The result from this projection is that a new image $\mathrm{R}(\mathrm{x}, \alpha)$, which is a sum of the intensities of the pixels in each direction, includes more geometric information than the original image. The concatenation of the Radon projections 
taken at $\theta=\mathrm{k} \pi / 6$ where $\mathrm{k}=0,1,2,3,4,5$ from local image was used to generate the feature vector. However, the Radon projections produce a high dimension feature vector, so that the PCA was applied to reduce the dimension. Finally, multiple linear regression (MLR) was applied for age learning. Local radon feature achieved a better result on FG-NET (6.18) than MORPH (6.65) and FERET (6.98)[59], which means that the radon feature work better on images with lower resolution [60].

Recently, there are different works that focus on wrinkles in age estimation algorithms [61], [62], [63], [44], [48], [64]. El-Dib and Onsi [42] investigated the ability of wrinkled appearance in eyes, the face parts excluding the forehead and the whole face to estimate the age. They found that the wrinkled appearance in eyes has a significant features related to the age, when it was compared with the other parts. In 2015, $\mathrm{Ng}$ et al. [44] investigated the influence of wrinkles on specific age estimation. They localized and presented the wrinkles in a significative way to estimate the age. They proposed a multiscale aging patterns (MAP) method, which extract the features directly from local patches. MAP was generated by applying a multi-scale filter to different face parts and wrinkles, and thus transformed into meaningful aging patterns. Wrinkles appear in a different sizes. To present this feature, MAP extends a method that used dynamic scale filter called Hybrid Hessian Filter (HHF), which is a wrinkle detection method proposed by $\mathrm{Ng}$ et al. [65]. Based on multi-level analysis of Hessian eigenvalues, the local behavior of the face image is emphasized and wrinkles were identified subsequently. Then a multi-scale aging patterns were constructed from extracted wrinkles. Finally, supervised learning algorithm was applied to learn and predict the age. This work demonstrated the ability of wrinkles to estimate the age on high resolution images, where the MAE is 4.87 on FERET [44].

Chang and Chen [43] introduced a framework for age estimation, which applied scattering transform to extract the features from face images and proposed a Cost-Sensitive Ordinal Hyperplanes Ranking (CSOHR) approach for age learning. Scattering Transform (ST) descriptor is a generalization of traditional BIF, where the first-layer of ST and BIF extract the features in a same way, except that the ST employed the Gaussian average in the pooling operators and BIF used standard deviation. ST provides a locally translation invariant representation, which linearizes small deformations, and provides co-occurrence coefficients which characterize textures. This feature is reduced in BIF by pooling operator. After extracting the features using ST, the ranking (or ordinal regression) method CSOHR that focus on pairwise ranking approach was used for age rank estimation. CSOHR with ST achieved 3.82 for MAE in MORPH album2 dataset and 4.70 in FG-NET with slower computation time than BIF for feature extraction and other Multiple Hyperplanes Ranker (MHR) for age learning. However, some features might be lost during dimensionality reduction process using PCA.

2) Global-Based Algorithms: Global features (also known as Holistic) are generally used to predict the age. It contain personal features such as gender, identity, ethnicity, and expression. Personal features are clearer in global features than aging features. Subspace learning techniques have been used extensively to extract global features. These include Locality Sensitive Discriminant Analysis (LSDA), Marginal Fisher Analysis (MFA) [39], PCA, Neighborhood Preserving Projections (NPP), Locality Preserving Projections (LPP), and Orthogonal LPP (OLPP) [31], [46]. However, Active Appearance Model (AAM) [88] is a most widely used method to extract global features for age estimation and to provide information about the shape and appearance of a face [40], [79].

AAM is a statistical appearance models proposed by Cootes (2001)[88]. It combines shape and texture variation. Building a face model require face images marked with points to determine the main features. The model apply Procrustes analysis to align the sets of points (represented as a vector) and build a statistical shape model. It then warps images by the mean shape, and normalized texture vector by applying a linear transformation. To build a texture model eigen-analysis was applied. Finally, a combined appearance model was generated by learning the correlations between shape and texture [88].

In 2002 AAM was introduced by Lanitis et al. [71] for first time for face age estimation. They generated statistical model from a set of training examples represented by a set of variables. They calculated the mean $\left(X_{m}\right)$ and standard deviation from training set, then applied principal components analysis (PCA) on covariance matrix of the deviation. Their experiment focus on age variation from childhood to adulthood (up to 30 years old). The images were aligned and warped to the mean shape to train shape and intensity model. Then the projection was used to define the aging variations using this formula: (age $=f(b)$ ). Three formulation were investigated for aging function (f): linear, quadratic and cubic. Based on their experiment, quadratic function achieved the best result [71].

Geng et al. [73] adopted AAM in their work. They developed an age estimation model known as AGES (AGing pattErn Subspace), which used a series of personal face images to model the aging process. An aging pattern can be defined as a sequence of personal face images sorted in time order, by learning a representative subspace. A projection was used to construct the aging pattern for unseen face image, which define the variation in the data set. While the position in aging pattern defines the specific age, the authors claimed that by using this feature the personalized characteristics were utilized well. An aging pattern can be completed if all positions in aging pattern are filled, otherwise it is called an incomplete aging. There are two stages in AGES: learning stage and age estimation. The aging pattern is highly incomplete, so that an iterative PCA was applied in learning stage to construct a representative subspace. The missing parts in the aging pattern were firstly initialized; and then the first subspace was constructed by applying standard PCA to the initialized data set. By solving a linear system, the subspace is used to reconstruct the missing parts, then the PCA is applied to update the aging pattern. These processes was repeated to minimize the reconstruction error. The age was estimated in two steps determine a suitable aging pattern from aging pattern subspace, and then find the specific age position within aging pattern [73]. However, the process of reconstruction of missing face images produces 
TABLE II

A SUMMARY OF FEATURE EXTRACTION METHODS.

\begin{tabular}{|c|c|c|}
\hline Feature Types & Year & Authors \\
\hline \multirow{11}{*}{ Local } & 1994 & Kwon and Lobo [51] \\
\hline & 2001 & Horng et al.[54] \\
\hline & 2006 & Ramanathan and Chellappa [55] \\
\hline & 2008 & Gunay et al. [66], Yan et al. [67] \\
\hline & 2009 & Gao et al. [68], Guo et al. [41] \\
\hline & 2011 & Choi et al. [62], El Dib and Onsi [42] \\
\hline & 2012 & Lin et al. [69] \\
\hline & 2013 & Ylioinas et al. [58], Gunay et al. [59] \\
\hline & 2014 & $\mathrm{Ng}$ et al. [48] \\
\hline & 2015 & $\begin{array}{l}\text { Ng et al. [44]. Dibeklioglu et al. [17], Chang et al. [43], Hadchum and Wongthanavasu } \\
\text { [63], Khan et al [64] }\end{array}$ \\
\hline & 2017 & Hsu et al. [70] \\
\hline \multirow{10}{*}{ Global } & 2002 & Lanitis et al. [71] \\
\hline & 2004 & Lanitis et al. [72] \\
\hline & 2006 & Ueki et al. [32] \\
\hline & 2007 & Geng et al. [73], Fu et al. [31] \\
\hline & 2008 & Guo et al. [46] \\
\hline & 2009 & Geng et al. [74], Geng and Kate [74], Luu et al. [75] \\
\hline & 2010 & Chang et al. [76], Zhang et al. [77] \\
\hline & 2011 & Chang et al. [78] \\
\hline & 2013 & Chao et al. [47] \\
\hline & 2016 & Bukar et al. [79] \\
\hline \multirow{8}{*}{ Hybrid } & 2008 & Suo et al $[80]$ \\
\hline & 2009 & Guo et al. [39] \\
\hline & 2010 & Luu et al. [81] \\
\hline & 2011 & Choi et al. [40], Guo et al. [82] \\
\hline & 2013 & Weng et al. [83], Tharwat et al. [19] \\
\hline & 2015 & Gunay and Nabiyev [84], Huerta et al. [85] \\
\hline & 2016 & Gunay and Nabiyev [86], Bekhouche et al. [49], Qiu [87] \\
\hline & 2017 & $\mathrm{Ng}$ et al. [60], [6] \\
\hline
\end{tabular}

a highly redundant face image, and this can increase the prediction error. In addition, the personalized method for age estimation such as AGES and methods in [77], [74], [71] ccan improve the performance of age estimation. However, this method is limited because it is difficult to acquire sequences of facial images for a single person during aging process [43].

Although AAM considers shape and texture features, it produces a high dimensional feature vector; and this may contain an outliers in the age labeling space that may not directly reflect the identical age labels [47]. Some valuable parts of the data (such as wrinkles) that represent discriminatory features might have been lost during dimensionality reduction process [48].

Fu et al. [31] adopted the Manifold Learning Model for first time to estimate the age. Generally, in age manifold, an aging pattern can be learned from many subjects at various ages. Thus, many personal faces are needed to represent ages as each person may have images at specific age or in an age range.
Age manifold use manifold embedding technique like OLPP to learn an aging pattern in low dimension from numerous face images at each age. In this model, each subject may have images only at one age in the database. The authors claimed that this feature makes manifold model more flexible and easier to collect a big aging face database [5]. By using this model to solve age estimation problem, the age manifold is learned to map the original data into a low-dimensional subspace after a pre-processing steps, in training phase. A regression function then was defined to fit the manifold data for further testing. In the testing phase, an input face image is transformed to the learning feature as in training phase. The age is predicted through the learned manifold mapping and regression model fitting. To learn a low-dimensional manifold in the embedded subspace of image space, a projection function which can be linear or nonlinear might use. This model compared between four different algorithms that use linear methods for projection to manifold space: LPP, OLPP, NPP, and PCA. They found 
that the OLPP achieved better performance than others. After finding the low dimensional manifold, an age estimation was defined as a multiple linear regression problem in the manifold space.

However, using manifold model required large size of training data to learn the embedded manifold [31], [5]. Another issue in this model is sensitivity to image mis-alignment caused by many factors, such as head rotation, different facial expression, and shape variations in facial growth and aging [14].

Luu et al. [75] introduced an age estimation technique using AAM and Support Vector Machines (SVMs). For each input face image I, 86 landmarks were determined and feature vector $x_{i}$ with size $30 \times 1$ was extracted using AAM. SVM was used to train a binary classification module, which consists of two parts: classifier $\mathrm{f}(\mathrm{x})$ to differentiate between youths $(0-20)$ and adults (21 to 69) and two aging functions: $f_{1}(x)$ as growth and development function and $f_{2}(x)$ as adult aging function. Finally, Support Vector Machine for regression (SVR) was used for age prediction. This algorithm outperforms the existing algorithms that used AAM by using the combination of binary classification and then age prediction.

In 2013 Chao et al. [47] adopted AAM for features extraction on their approach. After extracting feature using AAM, this approach added two steps: distance metric adjustment and dimensionality reduction to explore the relation between face features and age labels and mitigate the over-fitting problem in training the age prediction function. For this purpose, the Relevant Component Analysis (RCA) was applied for distance metric adjustment and LPP was used for dimensionality reduction. For age prediction, an age-oriented local regression named K Nearest Neighbors Support Vector Regression (KNN-SVR) was proposed. Although this approach achieved a good result, it used considerably additional functions which might increase the computational complexity.

In 2016 Bukar et al. [79] proposed supervised Appearance Model (sAM) for age and gender estimation that improves the AAM by using the partial least-squares (PLS) as the core of the model rather than PCA. They claimed that their proposed model (sAM) effectively represents the face features than AAM, whereas the PLS preserves worthy parts of the data that represent discriminatory features. PLS creates latent features via a linear combination of the predictor variables and response (class labels). Because sAM considers both shape and texture features, it was used as feature extractor in age estimation model, whereas ordinary least-square (OLS) and quadratic function $(\mathrm{QF})$ regressions were used for age learning with QF showing better results than OLS. The sAM model represents the faces in a convenient way to solve both age and gender classification problems, but it does not outperform the methods that used AAM.

3) Hybrid-Based Algorithms: In various face related applications, the local and global features were combined as hybrid features to offer superior performance. Choi et al. [40] introduced hybrid features representation and hierarchical classifier to increase the age estimation accuracy. For local features extraction, a set of region specific Gabor filters were used to extract wrinkle features. LBP was used for skin features, and
AAM was used for global features extraction. SVM and SVR were used for age classification and age regression. However, the combination of extracted features using this three methods (Gabor, LBP and AAM) produced a high dimension feature vector which can increase the computation time.

Guo et al. [82] also used hybrid features for age estimation. They extracted global features using Active Shape Model (ASM) and both Radon projection and 2D-DCT for local features, where the facial image is converted into Radon space then 2D-DCT is used to obtain the features from this space. Finally, SVM and SVR was used for age estimation. Their results showed that Radon-DCT achieves better results on FGNET, where the MAE is 4.18 years.

Gunay and Nabiyev [84], [86] conducted several experiments to estimate the age using different local and global methods to produce hybrid-based age estimation algorithms. In 2015 they proposed an age estimation method called "Global and Local feAture based Age estiMation (GLAAM)" that used AAM to extract global features and 2D-DCT for local features. PCA was applied to reduce the dimensionality after concatenating the local and global features. Finally, the age was estimated with multiple linear regression [84]. In 2016 Gunay and Nabiyev [86] repeated the experiment using different methods for local features. They applied Gabor filters to extract wrinkles and LBP for skin features, and applied PCA to reduce the dimensionality. Based on three types of features that were extracted (global, wrinkles and skin), Gunay and Nabiyev [86] modeled three aging functions separately with multiple linear regression, then they got the final decision by performing a decision level fusion to combine the results. They applied Gabor filter and LBP for local features to produce better result than 2D-DCT, where the MAE is 5.39 years for first experiment (AAM and 2D-DCT) and 4.87 years for second one (AAM, Gabor and LBP).

As mentioned above, wrinkle-based features are more effective for face representation. Based on this observation, and the fact that the hybrid based features can offering superior performance for age estimation, $\mathrm{Ng}$ et al. [60] proposed $\mathrm{Hy}-$ brid Ageing Patterns (HAP) feature representation to increase the age estimation accuracy. HAP combines the proposed Multi-scale Wrinkle Patterns (MWP) and Facial Appearance Model (FAM). MWP is a wrinkle-based feature representation method based on the wrinkle statistics, and used a robust wrinkle detection method called Hessian Line Tracking (HLT) [89] to extract wrinkle. The multi-scale wrinkles was extracted from ten regions: forehead, glabella, upper eyelids, crows feet (or eye corners), lower eyelids (or eyebag), cheeks, nasolabial grooves (or nasolabial folds), upper lips, marionette and lower lips. Then the wrinkle intensity and wrinkle density were calculated to represent the pattern. FAM is a generative parametric model that combined shape and texture features. PCA is used in FAM to reduce the dimension. After extracting the features using MWP and FAM the hybrid pattern was constructed. Finally, SVR was used to estimate age from the patterns. HAP outperform the existing method on FERET and MORPH, where the MAE is 3.02 and 3.68 respectively. However, HAP does not achieved a good result on FG-NET, because the texture information is unclear in FG-NET images. 
TABLE III

COMPARISON BETWEEN AGE LEARNING TECHNIQUES.

\begin{tabular}{|c|l|l|}
\hline Category & Strength & Weakness \\
\hline Classification & $\begin{array}{l}\text { More appropriate for age range } \\
\text { classification. }\end{array}$ & $\begin{array}{l}\text { Not appropriate to predict the age. } \\
\text { Class labels are uncorrelated. }\end{array}$ \\
\hline Regression & $\begin{array}{l}\text { Ability to predict age using a single } \\
\text { estimator. }\end{array}$ & $\begin{array}{l}\text { Does not consider the differences of age feature values accord- } \\
\text { ing to age groups. } \\
\text { It is difficult for regressor to learned a non-stationary kernels. }\end{array}$ \\
\hline Hierarchical & $\begin{array}{l}\text { The performance is improved by } \\
\text { considering the differences of age } \\
\text { feature values according to age } \\
\text { groups. }\end{array}$ & $\begin{array}{l}\text { The errors in the age group estimation step are propagated to the } \\
\text { specific age prediction step when using an age group estimator } \\
\text { with a hard boundary not including overlapped class. }\end{array}$ \\
\hline Ordinal ranking & $\begin{array}{l}\text { Preserve the relative order of age } \\
\text { labels and provides more stable in- } \\
\text { formation than exact age values. }\end{array}$ & Time consuming. \\
\hline Deep Learning & $\begin{array}{l}\text { Do not require predefined or hand } \\
\text { crafted features }\end{array}$ & Require large-scale data for training process. \\
\hline
\end{tabular}

Although the hybrid features can improve the performance of age estimation algorithm, it may increase the complexity and computation time for age estimation algorithms.

4) Summary: In general, different methods and algorithms can be effectively applied to estimate the age. Local features contains unique features that differentiate between age groups, so that it is suitable to handle age-group classification problem. On the other hand, individual characteristics might be more notable in global features, so that it is more suitable to predict the exact age. AAM can be recognized as a main model that extract the global feature, due to its ability to extract both shape and texture features. In hybrid features, Guo et al. [39] found that the performance of age estimation can be increased when combining BIF and manifold learning techniques. In addition to hybrid feature, using manifold learning techniques can reduce the dimensionality in BIF, while BIF addresses the problem of sensitivity to image mis-alignment in manifold learning techniques as it has the capability to deal with different changes in scale, translation and rotation.

More recently, researchers investigated the effectiveness of some appearance-based features and found that:

- Wrinkles can increase the age estimation accuracy [44], [60]. There is little research that focuses on age estimation based on wrinkles. However, most of them classified the age into groups rather than predict exact age.

- BIF, introduced by Guo et al. [41], achieved good results in age estimation. In 2017, Hsu et al. [70] proposed the Component Bio-Inspired Feature (CBIF) that achieved MAE of 3.38 on FG-NET.

\section{B. Age Learning Techniques}

As mentioned above, the existing facial age estimation methods consist of two phases: features extraction and age learning techniques. Once the features are extracted from the face images, learning technique is needed in order to classify or predict the age. In general, age learning techniques can be classified into three groups: age groups classification, regression or single-level age estimation and hierarchical age estimation [40]. Other techniques include ordinal ranking and deep learning. For clarification, It is noted that the machine learning techniques for classification, regression, hierarchical and ordinal ranking are referred to as conventional machine learning methods (non deep learning approaches). We summarised the strength and weakness of these five categories in Table III and a detailed review of these five categories.

1) Classification: Classification is an approach used to classify the age into multiple age groups such as babies, young, middle-aged adults, or old adults [51], [52], [54], [55], [53], [32], [72], [106]. This section review the classification methods based on conventional machine learning algorithms such as SVM [107], Linear Discriminant Analysis (LDA) [68], Artificial Neural Networks (ANN) [54], nearest neighbor classifier [72] or distance measure [66] for age group classification. Table IV provides some early popular works based on classification more than a decade ago. The most notable work was by Guo et al. [41] who achieved MAE of 4.77 using BIF features on FG-NET. It is noted that they used both classification and regression approaches to learn age.

$\mathrm{Ng}$ et al. [48] developed ANN with three layers: input, hidden and output to classify the age into two groups and applied their proposed method to investigate the effectiveness of wrinkles in age classification, which called Local Wrinklebased Extractor (LOWEX) that used Canny edge detection to detect the wrinkles from different face regions. Their proposed method with ANN achieved 80\% on FG-NET. Im et al. [108] extracted wrinkles feature using Gabor filter and applied SVM for age classification. Their experiment was tested on private data with average accuracy of $76.92 \%$. In 2017, Iqbal et al. [109] proposed local descriptor called Directional AgePrimitive Pattern (DAPP) and applied SVM to classify the age into 7 groups. They achieved accuracy of $73.1 \%$ on data collected from flickr.com.

In classification, the class labels are uncorrelated and each 
TABLE IV

COMPARISON BETWEEN DIFFERENT FACE AGE ESTIMATION ALGORITHMS ORGANISED BY MACHINE LEARNING TECHNIQUES AND DATABASE (PART I). TO CLARIFY, THE MACHINE LEARNING TECHNIQUE FOR CLASSIFICATION AND REGRESSION ARE REFERRED TO CONVENTIONAL MACHINE LEARNING ALGORITHMS

\begin{tabular}{|c|c|c|c|c|c|c|}
\hline $\begin{array}{l}\text { Machine } \\
\text { Learning } \\
\text { Technique }\end{array}$ & Database & Algorithm & $\begin{array}{c}\text { Feature Extraction } \\
\text { Method }\end{array}$ & MAE & $\begin{array}{c}\text { CS } \\
(0-10) \\
(\%)\end{array}$ & $\begin{array}{c}\text { Accuracy } \\
(\%)\end{array}$ \\
\hline \multirow{10}{*}{ Classification } & \multirow{5}{*}{ FG-NET } & Mohan et al. 2010 [90] & $\begin{array}{c}\text { Topological Texture } \\
\text { Features (TTF) }\end{array}$ & - & - & 94.1 \\
\hline & & Wang et al. 2010 [91] & ASM+BIF & - & - & 90 \\
\hline & & Liu et al. 2012 [92] & AAM & - & - & 79.2 \\
\hline & & Zheng et al. 2013[93] & LBP & - & - & 74.60 \\
\hline & & NG et al. 2014 [48] & LOWEX & - & - & 80 \\
\hline & \multirow[t]{2}{*}{ MORPH } & Zhou et al. 2011 [94] & Radon transform & - & - & $\sim 87$ \\
\hline & & Mirzaei et al. 2011 [95] & LBP & - & - & 67.23 \\
\hline & FERET & Gunay et al. 2008 [66] & LBP & - & - & 80 \\
\hline & \multirow[t]{2}{*}{ web data } & Gao et al. 2009 [68] & Gabor Filter & - & - & 91 \\
\hline & & Gao et al. 2009 [68] & LBP & - & - & 84.6 \\
\hline \multirow{11}{*}{ Regression } & \multirow{6}{*}{ FG-NET } & Geng et al. 2007 [73] & AGES & 6.77 & $\sim 81$ & - \\
\hline & & Guo et al. 2009 [41] & BIF & 4.77 & $\sim 90$ & - \\
\hline & & Chao et al. 2013 [47] & AAM & 4.38 & $\sim 90$ & - \\
\hline & & Gunay et al. 2015 [84] & AAM + 2D-DCT & 5.39 & - & - \\
\hline & & Wang et al. 2015 [96] & $\begin{array}{l}\text { deep learned aging } \\
\text { pattern (DLA) }\end{array}$ & 4.26 & $\sim 90$ & - \\
\hline & & $\mathrm{Ng}$ et al. 2017 [60] & HAP & 5.66 & - & - \\
\hline & \multirow[t]{2}{*}{ FERET } & $\mathrm{Ng}$ et al. 2015 [44] & $\begin{array}{l}\text { multi-scale aging } \\
\text { patterns (MAP) }\end{array}$ & 4.87 & - & - \\
\hline & & $\mathrm{Ng}$ et al. 2017 [60] & HAP & 3.02 & - & - \\
\hline & \multirow[t]{3}{*}{ MORPH } & Lu and Tan. 2010 [97] & $\begin{array}{l}\text { cost-sensitive locality } \\
\text { preserving projections } \\
\text { (CSLPP) }\end{array}$ & 4.24 & - & - \\
\hline & & Huerta et al. 2015 [85] & HOG + LBP + SURF & 4.25 & $\sim 90$ & - \\
\hline & & Ng et al. 2017 [60] & HAP & 3.68 & - & - \\
\hline
\end{tabular}

label is independent from each others. However, there is strong interrelationships between labels, and the age labels is wellordered in reality. For example, a person with 12 years old is more likely to be 11 or 13 years old. However, classification approaches do not reflect this property.

2) Regression: Regression approaches consider age label as a set of sequential numbers (e.g., 10, 11, 12,...). This sequence fits the ordinal nature of age label. This is a very popular age learning technique it includes many methods that can be used for regression such as SVR [46], Quadratic Regression [72], Multiple Linear Regression [84], and Multilayer Perceptron (MLP) [110]. Since 2007, Geng et al. [73] has achieved MAE of 6.77 years on FG-NET using facial appearance features. $\mathrm{Fu}$ et al. [31] applied multiple linear regression for age estimation after finding the low dimensional aging manifold using manifold embedding technique like OLPP. Later on, Guo et al. [46] used the manifold model to improve their work by using Locally Adjusted Robust Regression (LARR), which applied global and local steps respectively to learn the data. In the global learning, SVR was used for regression over all ages of the training data. Then a local adjustment within a limited range of ages centered at the regression result, where the linear SVM was used for local age adjustment. LARR produced MAE of 5.07 on FG-NET.

Guo et al. [39], [111] studied the effect of gender and race on age estimation. They applied different classifier and regressor to perform the gender, race and age estimation. However, these tasks were performed separately in different steps, where the gender or race classification was performed first, then the age was estimated within different class. To improve this work, in 2011, Guo et al. [112] proposed a kernel partial least squares (KPLS) regression algorithm for age estimation. KPLS have a flexible output vector that allows to overcome a number of related classification problems such as race, gender and age. It contains multiple labels in a same output vector. In addition, KPLS performs dimensionality reduction and learns the aging function in a single step. In this framework, KPLS was used to classify race, gender and estimate the age in single step. This age estimation method achieved MAE of 4.18 on MORPH.

In 2013, Chao et al. [47] proposed an age estimation algorithm using KNN-SVR. The authors claimed that the local regression function is more likely to overcome the problem of complicated facial aging. The MAE was 4.38 on FG-NET. 
TABLE V

COMPARISON BETWEEN DIFFERENT FACE AGE ESTIMATION ALGORITHMS ORGANISED BY MACHINE LEARNING TECHNIQUES AND DATABASE (PART II). THE MACHINE LEARNING TECHNIQUE FOR HIERARCHICAL AND ORDINAL RANKING ARE REFERRED TO CONVENTIONAL MACHINE LEARNING ALGORITHMS

\begin{tabular}{|c|c|c|c|c|c|c|}
\hline $\begin{array}{l}\text { Machine } \\
\text { Learning } \\
\text { Technique }\end{array}$ & Database & Algorithm & $\begin{array}{c}\text { Feature Extraction } \\
\text { Method }\end{array}$ & MAE & $\begin{array}{c}\text { CS } \\
(0-10) \\
(\%)\end{array}$ & $\begin{array}{c}\text { Accuracy } \\
(\%)\end{array}$ \\
\hline \multirow{11}{*}{ Hierarchical } & \multirow{6}{*}{ FG-NET } & Luu et al. 2010 [81] & $\begin{array}{l}\text { AAM + Local Ternary } \\
\text { Patterns (LTP) }\end{array}$ & 4.23 & $\sim 91$ & - \\
\hline & & Choi et al. 2011 [40] & $\begin{array}{c}\mathrm{AAM}+\underset{\text { Filter }}{\mathrm{LBP}}+\text { Gabor } \\
\end{array}$ & 4.65 & $\sim 991$ & - \\
\hline & & Li et al. 2012 [98] & $\mathrm{ASM}+\mathrm{LBP}$ & 4.32 & $\sim$ & - \\
\hline & & Han et al. 2013 [25] & BIF & 4.6 & - & - \\
\hline & & Hsu et al. 2017 [70] & CBIF & 3.38 & - & - \\
\hline & & Ren et al. 2014 [99] & $\begin{array}{c}\text { Scale Invariant Feature } \\
\text { Transform (SIFT) + } \\
\text { HOG + Gabor }\end{array}$ & 4.49 & - & - \\
\hline & \multirow{4}{*}{ MORPH } & Han et al. 2015 [24] & BIF & 3.6 & - & - \\
\hline & & Liu et al. 2015 & $\mathrm{BIF}+\mathrm{HOG}+\mathrm{LBP}$ & 2.97 & $\sim 95$ & - \\
\hline & & Pontes et al 2016 & $\begin{array}{c}\text { Local Phase } \\
\text { Quantization(LPQ) }\end{array}$ & 5.86 & $\sim 85$ & - \\
\hline & & Hsu et al. 2017 [70] & CBIF & 3.21 & - & - \\
\hline & PAL & Ren et al. 2014 [99] & SIFT + HOG + Gabor & 4.29 & - & - \\
\hline \multirow{6}{*}{$\begin{array}{l}\text { Ordinal } \\
\text { Ranking }\end{array}$} & \multirow{4}{*}{ FG-NET } & Yan et al. 2007 [100] & AGES & 5.33 & $\sim 85$ & - \\
\hline & & Chang et al. 2010 [76] & AAM & 5.79 & $\sim 85$ & - \\
\hline & & Chang et al. 2011 [78] & AAM & 4.48 & $\sim 90$ & - \\
\hline & & Liu et al. 2016 [101] & AAM & 4.14 & - & - \\
\hline & \multirow[t]{2}{*}{ MORPH } & Weng et al. 2013 [83] & $\mathrm{PCA}+\mathrm{LBP}+\mathrm{BIF}$ & 4.20 & $\sim 95$ & - \\
\hline & & Chang et al. 2015 [43] & ST & 3.82 & - & - \\
\hline \multirow{7}{*}{$\begin{array}{l}\text { Deep } \\
\text { Learning }\end{array}$} & \multirow{7}{*}{ MORPH } & Huerta et al. 2015 [85] & Deep Learning & 3.88 & - & 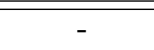 \\
\hline & & Niu et al. 2016 [102] & Deep Learning & 3.27 & $\sim 95$ & - \\
\hline & & Liu et al. 2016 [103] & ASM & 3.48 & - & - \\
\hline & & Niu et al. 2016 [102] & Deep Learning & 3.34 & $\sim 95$ & - \\
\hline & & Chen et al. 2017 [104] & Deep Learning & 2.96 & 97 & $\sim 97$ \\
\hline & & Liu et al. 2017 [105] & Deep Learning & 3.12 & $\sim 96$ & - \\
\hline & & Hsu et al. 2017 [70] & CBIF & 2.58 & - & - \\
\hline
\end{tabular}

Recently, Ng et al. [60] applied SVR with their proposed feature descriptor Hybrid Aging Patterns to estimate the age. They achieved a MAE of 3.02 on FERET. In 2017, Ng et al. [6] proposed a Multi-layer Age Regression (MAR) approach to estimate the age, consisting of two layers. Both layers produced a real number in output rather than class labels. FAM was used to extract the features in the first layer. FAM, BIF, KLBP and MWP were used to extract the features in second layer, then SVR was used for age prediction. Their experiment was validation on four dataset, i.e. FG-NET, MORPH, FERET and PAL. The best result was MAE of 3.00 on FERET. When compared this approach with LARR and Probabilistic Fusion Approach (PFA) [46], [113], PFA and LARR outperformed on FG-NET with the MAE of 4.97 and 5.07 respectively. PFA and LARR applied SVM rather than SVR in the second layer, and treat each age value in the age group as a class label.

Table IV summarises the research and validation mainly on three popular datasets (FG-NET, MORPH and FERET). The strength of regression is its ability to predict age using a single estimator. However, the personalized property in aging process produces non-stationary feature space, which is difficult for regressor to learn non-stationary kernels [78].

3) Hierarchical: Hierarchical age estimation combines the classification and regression methods to predict age. It provides more accurate result and simplifies the computational load, where the data was firstly classified into age groups, then the age was predicted from these pre-defined groups [40]. Choi et al. [40] applied age group classification followed by age regression to predict the age using SVM and SVR respectively. The hybrid features were extracted using AAM, LBP and Gabor. By using this combination of hybrid and hierarchical approach to predict the age, the MAE was 4.6 on FG-NET. Han et al. [25] also applied SVM and SVR, where SVM was used to classify the age into four groups after extracting features using BIF. Within each group, SVR was used to predict the age. In order to reduce the age classification error that produced from strict group boundaries, they used training samples for regression with distance up 
to 5 years between age groups. The MAE was 4.6 and 4.2 on FG-NET and MORPH respectively. Later, Han et al [24] improved their work and proposed a generic framework for gender, race and age estimation. In this framework, in order to fix the problem of low-quality images, they applied a quality assessment method to preprocess the images before extracting features. After preprocessing, the same steps and methods used in a previous work [25] were applied for classification and regression. The MAE was reduced by approximately 1 year for both datasets, with MAE of 3.8 on FG-NET and MAE of 3.6 on MORPH.

Li et al. [98] used Sparse Representation-based classification (SRC) algorithm to classify the age into two groups using the shape feature. The texture feature was concatenated with the shape feature and applied the Ordinal Hyperplane Ranker to estimate the age accurately. The MAE was 4.32 on FGNET. Angulu et al. [114] used ANN to classify the age into 6 groups then applied SVR to predict the age within these groups, after extracting the features using Multi-Frequency Biologically Inspired Features (MF-BIF). They achieved MAE of 4.02 on FG-NET. In 2017, Hsu et al. [70] performed the age classification and regression using traditional SVM and SVR after extracting the features using Component BioInspired Feature (CBIF). To reduce the classification error produced from strict age boundaries, they proposed moving segmentation method to find the age boundaries that can improve the accuracy. After applying the moving segmentation window, they found that the best age boundaries on FG-NET was at 11, 28 and 38 year, and the age boundaries on MORPH were at 29, 44 and 60 year. Based on their experiments, they found that the number of age groups in classification step have notable effect on the overall performance. The MAE was 3.38 on FG-NET and 3.21 on MORPH using 4 groups in classification step.

4) Ordinal ranking: Recently, there has been growing interest in age estimation to ordinal ranking (also called ordinal regression) due to the diversity in human aging processes at different age range. Hence, given two labels $x_{1}$ and $x_{2}$, the "greater than" information $\left(x_{2}>x_{1}\right.$ or $\left.x_{1}>x_{2}\right)$ is more reliable property for age estimation than the differences between the labels; and provides more stable information than exact age values. Ordinal ranking is built like when we ask a human "Who is the older between two people?". It seems easy to answer this question than asking people to predict the person's age. In ranking approach, the input face is compared with the known age faces from the database. This produces a series of comparisons. Age can then be estimated by integrating the results from these comparison. The age labels $l_{i}$ is treated as a rank order $l_{i} \in\{1, \ldots, x\}$ where $x$ is the labels number. Based on a given $x$, the dataset $\mathrm{D}$ was separated into two subsets $\left\{D_{x}^{+}\right.$and $\left.D_{x}^{-}\right\}$as in equation (3).

$$
\begin{aligned}
& D_{x}^{+}=\left(d_{i},+1\right) \mid l_{i}>x \\
& D_{x}^{-}=\left(d_{i},-1\right) \mid l_{i}<x
\end{aligned}
$$

These two subsets were used to learn the binary classifier and answer the query "Is the face older than age $x$ ?"; then making positive or negative decisions between the two sides (as a binary decision). By using these processes the age estimation task was reduced to a binary classification problem (any classification algorithm can be used as the binary classifier) at each query. Ordinal relationships between the age labels can then be recognized from a series of these query results.

Chang et al. [76] applied the ordinal ranker approach for age estimation in their work. They improved the work in [115], [76] and [116]. In [115] RankBoost algorithm applied cannot reflect the multiple thresholds of different classes properly due to the use of single hyperplane ranker in the feature space. On the other hand, parallel hyperplanes in [76] is more suitable when the database contains small number of classes. The RED-SVM used in [76] constructs $K-1$ parallel hyperplanes that separate the hyperspace into $K$ ranks by using $k-1$ thresholds. However, it is difficult to separate data using parallel hyperplanes when predicting the exact age as the $k$ is always large. The Multiple Hyperplanes Ranker used in [116] overlooks some potentially valuable cues provided by a well ordered set of labels. To overcome the limitations in previous work that used ranking approach, Chang et al. [76] introduced an ordinal hyperplanes ranker (OHRank), which aggregates $K-1$ binary classifiers based on the order of labels, after extracting the features using AAM. OHRank achieved 4.48 MAE on FG-NET.

5) Deep Learning: More recently, convolutional networks and deep learning approach have been successfully applied to several tasks related to facial analysis, including face alignment [117], face detection [118], face verification [119], facial skin analysis [7], and demographic estimation [120] to estimate the age and gender and to address face recognition problem. Later, many researchers implemented deep learning for age estimation [121], [122], [85], [123], [87], [124], [96], [125], [126], due to its superior performance over existing methods.

Generally, the basic methodology in deep learning approach is similar; it consists of alternating convolutional and pooling layers followed by fully-connected layers with the input to successive layers being the feature maps from previous layers. Weights in layers are changed simultaneously for representative features and classification with a specific loss function through back-propagation. The key of success in this approach is the accurate choice of parameters found in different layers [85]. However, deep learning requires a huge amount of data for training process.

Deep learning was introduced by Wang et al. [96] for a first time to solve age estimation problem. Their CNN architecture consists of three convolution layers, two pooling layers and full connection layer. Wang et al. [96] yield good results on FGNET and Morph. However, this CNN was used to represent the features then traditional linear SVR was applied for age prediction.

In 2016, Rothe et al. [124] proposed CNN called DEX based on VGG-16 architecture CNN [127]. VGG is much deeper CNN with small size for filters. DEX was pre-trained on the ImageNet [128] for image classification and fine-tune on the images from IMDB-WIKI dataset introduced by Rothe et al. [124]. Their experiments on MORPH yield MAE of 3.25. Rothe et al. [124] improves this result by fine-tuning the 


\section{NUMBER OF AGE ESTIMATION PUBLICATIONS BASED ON AGE LEARNING TECHNIQUES}

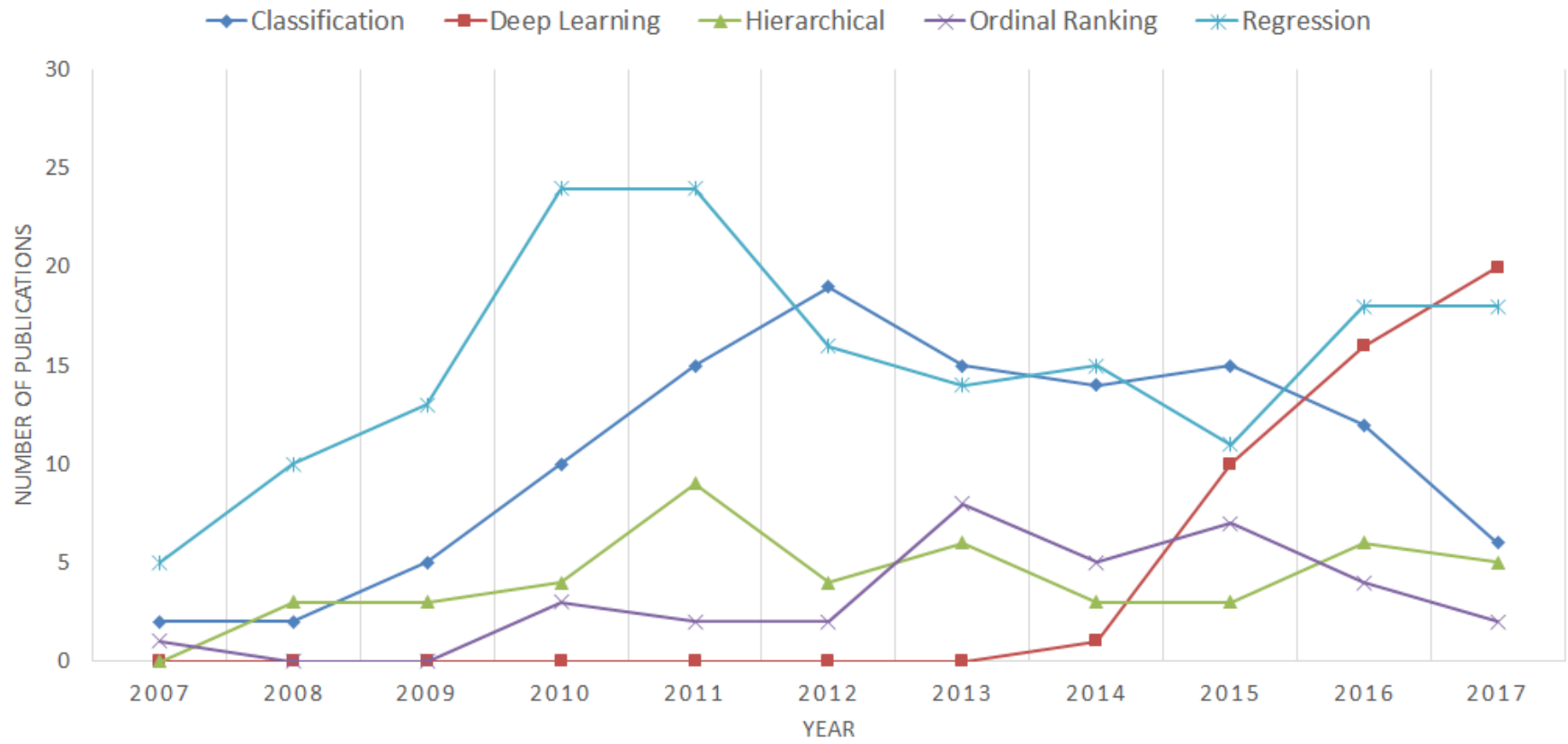

Fig. 2. Illustration of the age estimation publications based on five age learning techniques over the past 10 years. There was an increase of classification and regression methods until 2012. With the growth of deep learning, it is expected that the number of publications using deep learning has a rapid growth from 2014.

CNN on IMDB-WIKI before fine-tuning on the MORPH, this resulted MAE of 2.68 years. DEX achieved 4.63 on FG-NET without fine-tuning on IMDB-WIKI and 3.09 when fine-tuning on IMDB-WIKI before fine-tuning on FG-NET.

Niu et al. [102] proposed CNN called OR-CNN (Ordinal Ranking-CNN) to address age estimation problem consists of 3 convolutional, 3 normalization, and 2 pooling layers followed by a fully connected layer. They used binary ordinal age labels to train a series of basic CNNs, one for each age group. The binary results then are aggregated for the final age prediction. They claimed that the ranking method is preferred for age estimation as it produces smaller prediction error than softmax classifier,because softmax does not consider the ordinal relation between ages. Their experiment on MORPH achieved 3.27 years for MAE and 3.34 years using Asian face age dataset.

In 2017, Chen et al. [104] also proposed a Ranking Convolutional Neural Network (Ranking-CNN) framework. RankingCNN consists of three convolutional and sub-sampling layers, and three fully connected layers.. Ranking-CNN outperforms the existing methods for age estimation where the MAE is 2.69 years on MORPH. Table V summarises the deep learning methods on MORPH dataset. It is noted that the researchers preferred MORPH to FG-NET due to the dependency of deep learning on large-scale database.

Figure 2 compares the number of publications over the past decades based on our review. It illustrates the popularity of classification and regression techniques for age learning. However, from 2014, due to the rapid growth of deep learning, researchers started to explore and invest their time on deep learning technique.

6) Summary: In age learning, ordinal ranking and deep learning algorithms have recently demonstrated great success in handling age estimation problem. Ordinal ranking provides more stable information using a relative order of age labels. Due to the lack of face aging datasets, deep learning was used as features extractor (off the shelf CNN) [122], [96], [125] or fine-tuning the CNN with different datasets [121], [124], [123], [129].

\section{Conclusion And Future Directions}

A comprehensive survey on the state-of-the-art techniques for face age estimation was presented in this paper, including the new age learning technique that significantly improved the age estimation accuracy. In general, there are many factors that affected the overall performance of automatic age estimation, i.e. database, number of face images used for training, preprocessing and face alignment step, feature extraction method and the type of age learning technique.

In order to estimate the age accurately, the design of age estimation system requires careful selection of the face feature. To do so, Gunay et al. [84] stated some issues that need to take into consideration:

- Robust discriminative aging features extraction method is needed, to reduce the negative effect of individual variations.

- Face feature must be extracted from raw face images directly to speed up processing. 
- To facilitate the implementation of the classifiers, extracted face feature must lie in a low dimensional space.

- Minimization of the computational costs using suitable image size.

To conclude, we suggest some future directions for face age estimation as follow:

- Web collected images are an efficient way to collect a huge amount of images to establish face aging database, with balance in distribution of age, gender, ethnicity, expression, and pose. Developed web applications to collect images from internet [130] can facilitate the collection process. Large-scale database will have a positive impact on the performance of automatic age estimation system.

- Implementation of general framework that considers most of the factors affecting the performance of age estimation.

- In feature combination, finding a fusion strategy such as feature pooling [85] can produce more accurate results than just concatenating the features in hybrid approach.

- Combination or fusion of methods produces better result, e.g., combining wrinkle features with other local and global features [44], [60]. A similar strategy can be applied for scattering transform [43]. Combining deep learning, ordinal ranking and CBIF will produce superior result for age estimation. By combining these new techniques, the machine may outperforms the ability of human in face age estimation.

\section{REFERENCES}

[1] E. Meyers and L. Wolf, "Using biologically inspired features for face processing," International Journal of Computer Vision, vol. 76, no. 1, pp. 93-104, 2008.

[2] X. Han, M. H. Yap, and I. Palmer, "Face recognition in the presence of expressions," Journal of Software Engineering and Applications, vol. 5 , no. 05 , p. 321, 2012.

[3] N. Sarode and S. Bhatia, "Facial expression recognition," International Journal on computer science and Engineering, vol. 2, no. 5, pp. 1552 1557, 2010.

[4] Y. Wang, K. Ricanek, C. Chen, and Y. Chang, "Gender classification from infants to seniors," in Biometrics: theory applications and systems (BTAS), 2010 Fourth IEEE international conference on. IEEE, 2010, pp. 1-6.

[5] Y. Fu, G. Guo, and T. S. Huang, "Age synthesis and estimation via faces: A survey," Pattern Analysis and Machine Intelligence, IEEE Transactions on, vol. 32, no. 11, pp. 1955-1976, 2010.

[6] C.-C. Ng, Y.-T. Cheng, G.-S. Hsu, and M. H. Yap, "Multi-layer age regression for face age estimation," in Machine Vision Applications (MVA), 2017 Fifteenth IAPR International Conference on. IEEE, 2017, pp. 294-297.

[7] J. Alarifi, M. Goyal, A. Davison, D. Dancey, R. Khan, and M. H. Yap, "Facial skin classification using convolutional neural networks," in Image Analysis and Recognition: 14th International Conference, ICIAR 2017, Montreal, QC, Canada, July 5-7, 2017, Proceedings, vol. 10317. Springer, 2017, p. 479.

[8] O. F. Osman, R. M. I. Elbashir, I. E. Abbass, C. Kendrick, M. Goyal, and M. H. Yap, "Automated assessment of facial wrinkling: A case study on the effect of smoking," in 2017 IEEE International Conference on Systems, Man, and Cybernetics (SMC), Oct 2017, pp. 1081-1086.

[9] X. Geng, Y. Fu, and K. Smith-Miles, "Automatic facial age estimation," in 11th Pacific Rim International Conference on Artificial Intelligence, 2010, pp. 1-130.

[10] M. Y. M. H. El Dib, "Automatic facial age estimation," Ph.D. dissertation, Cairo University, 2011.

[11] "Japanese smokers to face age test." [Online]. Available: http: //news.bbc.co.uk/2/hi/asia-pacific/7395910.stm

[12] A. Dantcheva, P. Elia, and A. Ross, "What else does your biometric data reveal? a survey on soft biometrics," IEEE Transactions on Information Forensics and Security, vol. 11, no. 3, pp. 441-467, 2016.
[13] J. E. KLOEPPEL, "People who hope to keep their age a secret won't want to go near a computer running this software," 2010. [Online]. Available: https://news.illinois.edu/blog/view/6367/206178

[14] G. Guo, "Human age estimation and sex classification," in Video Analytics for Business Intelligence. Springer, 2012, pp. 101-131.

[15] K. Scherbaum, M. Sunkel, H.-P. Seidel, and V. Blanz, "Prediction of individual non-linear aging trajectories of faces," in Computer Graphics Forum, vol. 26, no. 3. Wiley Online Library, 2007, pp. 285-294.

[16] A. M. Albert, K. Ricanek, and E. Patterson, "A review of the literature on the aging adult skull and face: Implications for forensic science research and applications," Forensic Science International, vol. 172, no. 1, pp. 1-9, 2007.

[17] H. Dibeklioğlu, F. Alnajar, A. A. Salah, and T. Gevers, "Combining facial dynamics with appearance for age estimation," IEEE Transactions on Image Processing, vol. 24, no. 6, pp. 1928-1943, 2015.

[18] L. Boissieux, G. Kiss, N. M. Thalmann, and P. Kalra, "Simulation of skin aging and wrinkles with cosmetics insight," Computer Animation and Simulation 2000, pp. 15-27, 2000.

[19] A. Tharwat, A. M. Ghanem, and A. E. Hassanien, "Three different classifiers for facial age estimation based on k-nearest neighbor," in Computer Engineering Conference (ICENCO), 2013 9th International. IEEE, 2013, pp. 55-60.

[20] X. Wang, V. Ly, G. Lu, and C. Kambhamettu, "Can we minimize the influence due to gender and race in age estimation?" in Machine Learning and Applications (ICMLA), 2013 12th International Conference on, vol. 2. IEEE, 2013, pp. 309-314.

[21] C. Chen, A. Dantcheva, and A. Ross, "Impact of facial cosmetics on automatic gender and age estimation algorithms," in Computer Vision Theory and Applications (VISAPP), 2014 International Conference on, vol. 2. IEEE, 2014, pp. 182-190.

[22] Chen, Cunjian and Dantcheva, Antitza and Ross, Arun, "Automatic facial makeup detection with application in face recognition," in Biometrics (ICB), 2013 International Conference on. IEEE, 2013, pp. $1-8$.

[23] C. Bulpitt, H. Markowe, and M. Shipley, "Why do some people look older than they should?" Postgraduate medical journal, vol. 77, no. 911, pp. 578-581, 2001.

[24] H. Han, C. Otto, X. Liu, and A. K. Jain, "Demographic estimation from face images: Human vs. machine performance," IEEE transactions on pattern analysis and machine intelligence, vol. 37 , no. 6, pp. 1148 $1161,2015$.

[25] H. Han, C. Otto, and A. K. Jain, "Age estimation from face images: Human vs. machine performance," in Biometrics (ICB), 2013 International Conference on. IEEE, 2013, pp. 1-8.

[26] B.-C. Chen, C.-S. Chen, and W. H. Hsu, "Cross-age reference coding for age-invariant face recognition and retrieval," in European Conference on Computer Vision. Springer, 2014, pp. 768-783.

[27] P. J. Phillips, P. J. Rauss, S. Z. Der et al., FERET (face recognition technology) recognition algorithm development and test results. Army Research Laboratory Adelphi, MD, 1996.

[28] FGNET aging dataset, http://www-prima.inrialpes.fr/FGnet/, accessed on September 2012.

[29] K. Ricanek Jr and T. Tesafaye, "Morph: A longitudinal image database of normal adult age-progression," in Automatic Face and Gesture Recognition, 2006. FGR 2006. 7th International Conference on. IEEE, 2006, pp. 341-345.

[30] M. Minear and D. C. Park, "A lifespan database of adult facial stimuli," Behavior Research Methods, Instruments, \& Computers, vol. 36, no. 4, pp. 630-633, 2004.

[31] Y. Fu, Y. Xu, and T. S. Huang, "Estimating human age by manifold analysis of face pictures and regression on aging features," in Multimedia and Expo, 2007 IEEE International Conference on. IEEE, 2007, pp. 1383-1386.

[32] K. Ueki, T. Hayashida, and T. Kobayashi, "Subspace-based age-group classification using facial images under various lighting conditions," in Automatic Face and Gesture Recognition, 2006. FGR 2006. 7th International Conference on. IEEE, 2006, pp. 6-pp.

[33] Y. Fu and N. Zheng, "M-face: An appearance-based photorealistic model for multiple facial attributes rendering," Circuits and Systems for Video Technology, IEEE Transactions on, vol. 16, no. 7, pp. 830842,2006

[34] H. Fukai, H. Takimoto, M. Fukumi, and Y. Mitsukura, Apparent age estimation system based on age perception. INTECH Open Access Publisher, 2011.

[35] A. Bastanfard, M. A. Nik, and M. M. Dehshibi, "Iranian face database with age, pose and expression," in Machine Vision, 2007. ICMV 2007. International Conference on. IEEE, 2007, pp. 50-55. 
[36] J. Suo, F. Min, S. Zhu, S. Shan, and X. Chen, "A multi-resolution dynamic model for face aging simulation," in Computer Vision and Pattern Recognition, 2007. CVPR'07. IEEE Conference on. IEEE, 2007, pp. 1-8.

[37] B. Ni, Z. Song, and S. Yan, "Web image mining towards universal age estimator," in Proceedings of the 17th ACM international conference on Multimedia. ACM, 2009, pp. 85-94.

[38] "Morph database." [Online]. Available: http://www.faceaginggroup. $\mathrm{com} / \mathrm{morph} /$

[39] G. Guo, G. Mu, Y. Fu, C. R. Dyer, and T. S. Huang, "A study on automatic age estimation using a large database," in ICCV, 2009, pp. 1986-1991.

[40] S. E. Choi, Y. J. Lee, S. J. Lee, K. R. Park, and J. Kim, "Age estimation using a hierarchical classifier based on global and local facial features," Pattern Recognition, vol. 44, no. 6, pp. 1262-1281, 2011.

[41] G. Guo, G. Mu, Y. Fu, and T. S. Huang, "Human age estimation using bio-inspired features," in Computer Vision and Pattern Recognition, 2009. CVPR 2009. IEEE Conference on. IEEE, 2009, pp. 112-119.

[42] M. Y. El Dib and H. M. Onsi, "Human age estimation framework using different facial parts," Egyptian Informatics Journal, vol. 12, no. 1, pp. 53-59, 2011.

[43] K.-Y. Chang and C.-S. Chen, "A learning framework for age rank estimation based on face images with scattering transform," IEEE Transactions on Image Processing, vol. 24, no. 3, pp. 785-798, 2015.

[44] C.-C. Ng, M. H. Yap, N. Costen, and B. Li, "Will wrinkle estimate the face age?" in Systems, Man, and Cybernetics (SMC), 2015 IEEE International Conference on. IEEE, 2015, pp. 2418-2423.

[45] D. T. Nguyen, S. R. Cho, K. Y. Shin, J. W. Bang, and K. R. Park, "Comparative study of human age estimation with or without preclassification of gender and facial expression," The Scientific World Journal, vol. 2014, 2014.

[46] G. Guo, Y. Fu, C. R. Dyer, and T. S. Huang, "Image-based human age estimation by manifold learning and locally adjusted robust regression," IEEE Transactions on Image Processing, vol. 17, no. 7, pp. 1178-1188, 2008.

[47] W.-L. Chao, J.-Z. Liu, and J.-J. Ding, "Facial age estimation based on label-sensitive learning and age-oriented regression," Pattern Recognition, vol. 46, no. 3, pp. 628-641, 2013.

[48] C.-C. Ng, M. H. Yap, N. Costen, and B. Li, "An investigation on local wrinkle-based extractor of age estimation," in Computer Vision Theory and Applications (VISAPP), 2014 International Conference on, vol. 1. IEEE, 2014, pp. 675-681.

[49] S. E. Bekhouche, A. Ouafi, A. Taleb-Ahmed, A. Hadid, and A. Benlamoudi, "Facial age estimation using bsif and lbp," arXiv preprint arXiv:1601.01876, 2016.

[50] Y. Su, S. Shan, X. Chen, and W. Gao, "Hierarchical ensemble of global and local classifiers for face recognition," Image Processing, IEEE Transactions on, vol. 18, no. 8, pp. 1885-1896, 2009.

[51] Y. H. Kwon and N. D. V. Lobo, "Age classification from facial images," in Computer Vision and Pattern Recognition, 1994. Proceedings CVPR'94., 1994 IEEE Computer Society Conference on. IEEE, 1994, pp. 762-767.

[52] Y. H. Kwon and N. da Vitoria Lobo, "Age classification from facial images," Computer Vision and Image Understanding, vol. 74, no. 1, pp. 1-21, 1999.

[53] J.-G. Wang, W.-Y. Yau, and H. L. Wang, "Age categorization via ecoc with fused gabor and lbp features," in Applications of Computer Vision (WACV), 2009 Workshop on. IEEE, 2009, pp. 1-6.

[54] W.-B. Horng, C.-P. Lee, and C.-W. Chen, "Classification of age groups based on facial features," Tamkang Journal of Science and Engineering, vol. 4, no. 3, pp. 183-192, 2001.

[55] N. Ramanathan and R. Chellappa, "Modeling age progression in young faces," in Computer Vision and Pattern Recognition, 2006 IEEE Computer Society Conference on, vol. 1. IEEE, 2006, pp. 387-394.

[56] M. Riesenhuber and T. Poggio, "Hierarchical models of object recognition in cortex," Nature neuroscience, vol. 2, no. 11, pp. 1019-1025, 1999.

[57] T. Serre, L. Wolf, S. Bileschi, M. Riesenhuber, and T. Poggio, "Robust object recognition with cortex-like mechanisms," Pattern Analysis and Machine Intelligence, IEEE Transactions on, vol. 29, no. 3, pp. 411426, 2007.

[58] J. Ylioinas, A. Hadid, X. Hong, and M. Pietikäinen, "Age estimation using local binary pattern kernel density estimate," in International Conference on Image Analysis and Processing. Springer, 2013, pp. 141-150.
[59] A. Günay and V. V. Nabiyev, "Age estimation based on local radon features of facial images," in Computer and Information Sciences III. Springer, 2013, pp. 183-190.

[60] C.-C. Ng, M. H. Yap, Y.-T. Cheng, and G.-S. Hsu, "Hybrid ageing patterns for face age estimation," Image and Vision Computing, 2017.

[61] J. Aznar-Casanova, N. Torro-Alves, and S. Fukusima, "How much older do you get when a wrinkle appears on your face? modifying age estimates by number of wrinkles," Aging, Neuropsychology, and Cognition, vol. 17, no. 4, pp. 406-421, 2010.

[62] Y.-H. Choi, Y.-S. Tak, S. Rho, and E. Hwang, "Accurate wrinkle representation scheme for skin age estimation," in Multimedia and Ubiquitous Engineering (MUE), 2011 5th FTRA International Conference on. IEEE, 2011, pp. 226-231.

[63] P. Hadchum and S. Wongthanavasu, "Facial age estimation using a hybrid of svm and fuzzy logic," in Electrical Engineering/Electronics, Computer, Telecommunications and Information Technology (ECTICON), 2015 12th International Conference on. IEEE, 2015, pp. 1-6.

[64] S. Khan, S. Khan, T. Khan, A. Hussain, A. Siddique, and N. Ahmad, "Wrinkles energy based age estimation using discrete cosine transform," in Emerging Technologies (ICET), 2015 International Conference on. IEEE, 2015, pp. 1-4.

[65] C.-C. Ng, M. H. Yap, N. Costen, and B. Li, "Automatic wrinkle detection using hybrid hessian filter," in Asian Conference on Computer Vision. Springer, 2014, pp. 609-622.

[66] A. Günay and V. V. Nabiyev, "Automatic age classification with lbp," in Computer and Information Sciences, 2008. ISCIS'08. 23rd International Symposium on. IEEE, 2008, pp. 1-4.

[67] S. Yan, M. Liu, and T. S. Huang, "Extracting age information from local spatially flexible patches," in Acoustics, Speech and Signal Processing, 2008. ICASSP 2008. IEEE International Conference on. IEEE, 2008, pp. 737-740

[68] F. Gao and H. Ai, "Face age classification on consumer images with gabor feature and fuzzy lda method," in Advances in biometrics. Springer, 2009, pp. 132-141.

[69] C.-T. Lin, D.-L. Li, J.-H. Lai, M.-F. Han, and J.-Y. Chang, "Automatic age estimation system for face images," International Journal of Advanced Robotic Systems, vol. 29, 2012.

[70] G.-S. Jison Hsu, Y.-T. Cheng, C. Ching Ng, and M. Hoon Yap, "Component biologically inspired features with moving segmentation for age estimation," in Proceedings of the IEEE Conference on Computer Vision and Pattern Recognition Workshops, 2017, pp. 38-45.

[71] A. Lanitis, C. J. Taylor, and T. F. Cootes, "Toward automatic simulation of aging effects on face images," Pattern Analysis and Machine Intelligence, IEEE Transactions on, vol. 24, no. 4, pp. 442-455, 2002.

[72] A. Lanitis, C. Draganova, and C. Christodoulou, "Comparing different classifiers for automatic age estimation," Systems, Man, and Cybernetics, Part B: Cybernetics, IEEE Transactions on, vol. 34, no. 1, pp. 621-628, 2004.

[73] X. Geng, Z.-H. Zhou, and K. Smith-Miles, "Automatic age estimation based on facial aging patterns," Pattern Analysis and Machine Intelligence, IEEE Transactions on, vol. 29, no. 12, pp. 2234-2240, 2007.

[74] X. Geng and K. Smith-Miles, "Facial age estimation by multilinear subspace analysis," in Acoustics, Speech and Signal Processing, 2009. ICASSP 2009. IEEE International Conference on. IEEE, 2009, pp. 865-868

[75] K. Luu, K. Ricanek Jr, T. D. Bui, and C. Y. Suen, "Age estimation using active appearance models and support vector machine regression," in Biometrics: Theory, Applications, and Systems, 2009. BTAS'09. IEEE 3rd International Conference on. IEEE, 2009, pp. 1-5.

[76] K.-Y. Chang, C.-S. Chen, and Y.-P. Hung, "A ranking approach for human ages estimation based on face images," in Pattern Recognition (ICPR), 2010 20th International Conference on. IEEE, 2010, pp. 3396-3399.

[77] Y. Zhang and D.-Y. Yeung, "Multi-task warped gaussian process for personalized age estimation," in Computer Vision and Pattern Recognition (CVPR), 2010 IEEE Conference on. IEEE, 2010, pp. 2622-2629.

[78] K.-Y. Chang, C.-S. Chen, and Y.-P. Hung, "Ordinal hyperplanes ranker with cost sensitivities for age estimation," in Computer vision and pattern recognition (cvpr), 2011 ieee conference on. IEEE, 2011, pp. 585-592.

[79] A. M. Bukar, H. Ugail, and D. Connah, "Automatic age and gender classification using supervised appearance model," Journal of Electronic Imaging, vol. 25, no. 6, pp. 061 605-061 605, 2016.

[80] J. Suo, T. Wu, S. Zhu, S. Shan, X. Chen, and X. Gao, "Design sparse features for age estimation using hierarchical face model," in Automatic 
Face \& Gesture Recognition, 2008. FG'08. 8th IEEE International Conference on. IEEE, 2008, pp. 1-6.

[81] K. Luu, T. D. Bui, C. Y. Suen, and K. Ricanek Jr, "Combined local and holistic facial features for age-determination," in Control Automation Robotics \& Vision (ICARCV), 2010 11th International Conference on. IEEE, 2010, pp. 900-904.

[82] J.-M. Guo, Y.-M. Liou, and H.-S. Nguyen, "Human face age estimation with adaptive hybrid features," in System Science and Engineering (ICSSE), 2011 International Conference on. IEEE, 2011, pp. 55-58.

[83] R. Weng, J. Lu, G. Yang, and Y.-P. Tan, "Multi-feature ordinal ranking for facial age estimation," in Automatic face and gesture recognition (FG), 2013 10th IEEE international conference and workshops on. IEEE, 2013, pp. 1-6.

[84] A. Günay and V. V. Nabiyev, "Age estimation based on aam and 2d-dct features of facial images," International Journal of Computer Science and Applications, vol. 6, no. 2, 2015.

[85] I. Huerta, C. Fernández, C. Segura, J. Hernando, and A. Prati, "A deep analysis on age estimation," Pattern Recognition Letters, vol. 68, pp. 239-249, 2015.

[86] A. Günay and V. V. Nabiyev, "Age estimation based on hybrid features of facial images," in Information Sciences and Systems 2015. Springer, 2016, pp. 295-304.

[87] J. Qiu et al., "Convolutional neural network based age estimation from facial image and depth prediction from single image," 2016.

[88] T. F. Cootes, G. J. Edwards, and C. J. Taylor, "Active appearance models," IEEE Transactions on Pattern Analysis \& Machine Intelligence, no. 6, pp. 681-685, 2001.

[89] C.-C. Ng, M. H. Yap, N. Costen, and B. Li, "Wrinkle detection using hessian line tracking," IEEE Access, vol. 3, pp. 1079-1088, 2015.

[90] M. C. Mohan, V. Vijaya Kumar, and V. Venkata Krishna, "Novel method of adult age classification using linear wavelet transforms," IJCSNS, vol. 10, no. 3, p. 61, 2010.

[91] S. Wang, X. Xia, J. Le, S. Yang, and X. Liao, "Classifying children's and adults' faces by bio-inspired features," in Intelligent Computing and Intelligent Systems (ICIS), 2010 IEEE International Conference on, vol. 3. IEEE, 2010, pp. 673-677.

[92] L. Liu, J. Liu, and J. Cheng, "Age-group classification of facial images," in Machine Learning and Applications (ICMLA), 2012 11th International Conference on, vol. 1. IEEE, 2012, pp. 693-696.

[93] Y. Zheng, H. Yao, Y. Zhang, and P. Xu, "Age classification based on back-propagation network," in Proceedings of the Fifth International Conference on Internet Multimedia Computing and Service. ACM, 2013, pp. 319-322.

[94] H. Zhou, P. C. Miller, and J. Zhang, "Age classification using radon transform and entropy based scaling svm." in BMVC, 2011, pp. 1-12.

[95] F. Mirzaei and Ö. Toygar, "Facial age classification using subpatternbased approaches," studies, vol. 2, no. 5, p. 15, 2011.

[96] X. Wang, R. Guo, and C. Kambhamettu, "Deeply-learned feature for age estimation," in 2015 IEEE Winter Conference on Applications of Computer Vision (WACV). IEEE, 2015, pp. 534-541.

[97] J. Lu and Y.-P. Tan, "Cost-sensitive subspace learning for face recognition," in Computer Vision and Pattern Recognition (CVPR), 2010 IEEE Conference on. IEEE, 2010, pp. 2661-2666.

[98] W. Li, Y. Wang, and Z. Zhang, "A hierarchical framework for image-based human age estimation by weighted and ohranked sparse representation-based classification," in Biometrics (ICB), 2012 5th IAPR International Conference on. IEEE, 2012, pp. 19-25.

[99] H. Ren and Z.-N. Li, "Age estimation based on complexity-aware features," in Asian Conference on Computer Vision. S Springer, 2014, pp. $115-128$.

[100] S. Yan, H. Wang, T. S. Huang, Q. Yang, and X. Tang, "Ranking with uncertain labels," in Multimedia and Expo, 2007 IEEE International Conference on. IEEE, 2007, pp. 96-99.

[101] H. Liu and X. Sun, "A partial least squares based ranker for fast and accurate age estimation," in Acoustics, Speech and Signal Processing (ICASSP), 2016 IEEE International Conference on. IEEE, 2016, pp. 2792-2796.

[102] Z. Niu, M. Zhou, L. Wang, X. Gao, and G. Hua, "Ordinal regression with multiple output cnn for age estimation," in Proceedings of the IEEE Conference on Computer Vision and Pattern Recognition, 2016, pp. 4920-4928.

[103] T.-J. Liu, K.-H. Liu, H.-H. Liu, and S.-C. Pei, "Age estimation via fusion of multiple binary age grouping systems," in Image Processing (ICIP), 2016 IEEE International Conference on. IEEE, 2016, pp. 609-613.
[104] S. Chen, C. Zhang, M. Dong, J. Le, and M. Rao, "Using rankingcnn for age estimation," in Proceedings of the IEEE Conference on Computer Vision and Pattern Recognition, 2017, pp. 5183-5192.

[105] H. Liu, J. Lu, J. Feng, and J. Zhou, "Ordinal deep feature learning for facial age estimation," in Automatic Face \& Gesture Recognition (FG 2017), 2017 12th IEEE International Conference on. IEEE, 2017, pp. 157-164.

[106] I. Bouchrika, A. Ladjailia, N. Harrati, and S. Khedairia, "Automated clustering and estimation of age groups from face images using the local binary pattern operator," in Electrical Engineering (ICEE), 2015 4th International Conference on. IEEE, 2015, pp. 1-4.

[107] H.-C. Lian and B.-L. Lu, "Age estimation using a min-max modular support vector machine," in Twelfth International Conference on Neural Information Processing, 2005, pp. 83-88.

[108] S. mi Im, H. yeon Cho, and T. Kim, "Age estimation based on facial wrinkles by using the gabor filter and svm," TECHART: Journal of Arts and Imaging Science, vol. 3, no. 4, pp. 24-26, 2016.

[109] M. T. B. Iqbal, M. Shoyaib, B. Ryu, M. Abdullah-Al-Wadud, and O. Chae, "Directional age-primitive pattern (dapp) for human age group recognition and age estimation," IEEE Transactions on Information Forensics and Security, 2017.

[110] M. A. Jadid and O. S. Sheijani, "Facial age estimation under the terms of local latency using weighted local binary pattern and multi-layer perceptron," in Control, Instrumentation, and Automation (ICCIA), 2016 4th International Conference on. IEEE, 2016, pp. 184-189.

[111] G. Guo and G. Mu, "Human age estimation: What is the influence across race and gender?" in Computer Vision and Pattern Recognition Workshops (CVPRW), 2010 IEEE Computer Society Conference on. IEEE, 2010, pp. 71-78.

[112] Guo, Guodong and Mu, Guowang, "Simultaneous dimensionality reduction and human age estimation via kernel partial least squares regression," in Computer vision and pattern recognition (cvpr), 2011 ieee conference on. IEEE, 2011, pp. 657-664.

[113] G. Guo, Y. Fu, C. R. Dyer, and T. S. Huang, "A probabilistic fusion approach to human age prediction," in Computer Vision and Pattern Recognition Workshops, 2008. CVPRW'08. IEEE Computer Society Conference on. IEEE, 2008, pp. 1-6.

[114] R. Angulu, J. R. Tapamo, and A. O. Adewumi, "Human age estimation using multi-frequency biologically inspired features (mf-bif)," in AFRICON, 2017 IEEE. IEEE, 2017, pp. 26-31.

[115] P. Yang, L. Zhong, and D. Metaxas, "Ranking model for facial age estimation," in Pattern Recognition (ICPR), 2010 20th International Conference on. IEEE, 2010, pp. 3404-3407.

[116] T. Qin, X.-D. Zhang, D.-S. Wang, T.-Y. Liu, W. Lai, and H. Li, "Ranking with multiple hyperplanes," in Proceedings of the 30th annual international ACM SIGIR conference on Research and development in information retrieval. ACM, 2007, pp. 279-286.

[117] Z. Zhang, P. Luo, C. C. Loy, and X. Tang, "Learning deep representation for face alignment with auxiliary attributes," IEEE transactions on pattern analysis and machine intelligence, vol. 38, no. 5, pp. 918-930, 2016.

[118] Y. Sun, X. Wang, and X. Tang, "Deep convolutional network cascade for facial point detection," in Proceedings of the IEEE conference on computer vision and pattern recognition, 2013, pp. 3476-3483.

[119] Y. Taigman, M. Yang, M. Ranzato, and L. Wolf, "Closing the gap to human-level performance in face verification. deepface," in IEEE Computer Vision and Pattern Recognition (CVPR), 2014.

[120] M. Yang, S. Zhu, F. Lv, and K. Yu, "Correspondence driven adaptation for human profile recognition," in Computer Vision and Pattern Recognition (CVPR), 2011 IEEE Conference on. IEEE, 2011, pp. 505-512.

[121] Y. Dong, Y. Liu, and S. Lian, "Automatic age estimation based on deep learning algorithm," Neurocomputing, vol. 187, pp. 4-10, 2016.

[122] F. Gurpinar, H. Kaya, H. Dibeklioglu, and A. Salah, "Kernel elm and cnn based facial age estimation," in Proceedings of the IEEE Conference on Computer Vision and Pattern Recognition Workshops, 2016, pp. 80-86.

[123] R. C. Malli, M. Aygun, and H. K. Ekenel, "Apparent age estimation using ensemble of deep learning models," arXiv preprint arXiv:1606.02909, 2016.

[124] R. Rothe, R. Timofte, and L. Van Gool, "Deep expectation of real and apparent age from a single image without facial landmarks," International Journal of Computer Vision, pp. 1-14, 2016.

[125] D. Zheng, J. Du, W. Fan, J. Wang, and C. Zhai, "Deep learning with pcanet for human age estimation," in International Conference on Intelligent Computing. Springer, 2016, pp. 300-310. 
[126] Y. Zhu, Y. Li, G. Mu, and G. Guo, "A study on apparent age estimation," in Proceedings of the IEEE International Conference on Computer Vision Workshops, 2015, pp. 25-31.

[127] K. Simonyan and A. Zisserman, "Very deep convolutional networks for large-scale image recognition," arXiv preprint arXiv:1409.1556, 2014.

[128] O. Russakovsky, J. Deng, H. Su, J. Krause, S. Satheesh, S. Ma Z. Huang, A. Karpathy, A. Khosla, M. Bernstein et al., "Imagenet large scale visual recognition challenge," International Journal of Computer Vision, vol. 115, no. 3, pp. 211-252, 2015.

[129] R. Rothe, R. Timofte, and L. Van Gool, "Deep expectation of real and apparent age from a single image without facial landmarks," International Journal of Computer Vision, vol. 126, no. 2-4, pp. 144 157, 2018.

[130] S. Escalera, J. Gonzalez, X. Baró, P. Pardo, J. Fabian, M. Oliu, H. J. Escalante, I. Huerta, and I. Guyon, "Chalearn looking at people 2015 new competitions: Age estimation and cultural event recognition," in Neural Networks (IJCNN), 2015 International Joint Conference on. IEEE, 2015, pp. 1-8.

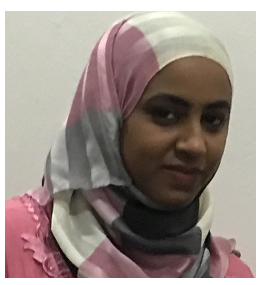

Omaima FathEIrahman Osman received her BSc degree in Computer and Information System from Sudan University of Science and Technology (SUST), Sudan in 2009. She received the MSc degree in Computer Science from Khartoum University in 2012. She is now a PhD student at SUST. She is a Lecturer in school of Computer Science at Sharq Elniel College. Her research interests include face analysis and pattern recognition.

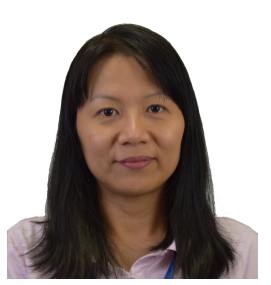

Moi Hoon Yap is a Reader in Computer Science at the Manchester Metropolitan University and a Royal Society Industry Fellow with Image Metrics Ltd. She received her $\mathrm{PhD}$ in Computer Science from Loughborough University in 2009. After her PhD, she worked as Postdoctoral Research Assistant (April 09 - Oct 11) in the Centre for Visual Computing at the University of Bradford. She serves as an Associate Editor for Journal of Open Research Software and reviewers for IEEE transactions/journals (Image Processing, Multimedia, Cybernetics, biomedical health and informatics). Her research expertise is computer vision, applied machine learning and deep learning. 